\title{
Engineered nanostructures to carry the biological ligands
}

\author{
Subash C.B. Gopinath ${ }^{1,2^{*}}$, Santheraleka Ramanathan $^{1}$, Koh Hann Suk ${ }^{1}$, Mu Ee Foo ${ }^{1}$, Periasamy Anbu ${ }^{3}$, and M.N.A. Uda ${ }^{1}$ \\ ${ }^{1}$ School of Bioprocess Engineering, Universiti Malaysia Perlis, 02600 Arau, Perlis, Malaysia. \\ ${ }^{2}$ Institute of Nano Electronic Engineering, Universiti Malaysia Perlis, 01000 Kangar, Perlis, Malaysia. \\ ${ }^{3}$ Department of Biological Engineering, College of Engineering, Inha University, Incheon 402-751, Republic of Korea.
}

\begin{abstract}
Different nanostructures were engineered with the nanoscale dimension lesser than $100 \mathrm{~nm}$. These nanostructures include silver, cellulose nanoparticles and single-walled carbon nanotube (SWCNT). Biological ligands were obtained from the medicinally important herbal plants, such as Solanum trilobatum and Hempedu bumi and conjugated with the nanostructures silver nanoparticle and SWCNT, respectively. On the other hand, bio-ligands from cow urine were encapsulated in the cellulose nanoparticle. To confirm morphology these nanostructures, they were observed under Field Emission Scanning Electron Microscope and the results displayed the uniformed nanostructures. Further, biological ligand carrying ability of these nanostructures was confirmed by the bacterial inhibition assay on the agar plate. This study provided the evidence on the capability of nanostructures to carry the biological ligands.
\end{abstract}

\section{Introduction}

Currently, nanotechnology is a booming discipline implemented effectively in various sectors. The development of high-performance nanomaterials could be a central importance for medical diagnosis, target validation and drug-delivery [1-6]. Careful selection of proper nanomaterials is aiding to interacting biomolecules for various downstream applications. Success in the massive production of nanomaterials is mainly driven by top-down and bottom-up approaches and making sequential alignments among interdisciplinary sciences [7]. Under laboratory scale, various nanomaterials were fabricated with the aim to bridge the industries. There are many researches have been carried out on engineering the nanoparticles. The obtained particle size plays an importance role in many sectors, due to their nanoscale in dimension. There are many methods can be used to test the toxicity of engineering nanoparticles towards microorganisms which display the antimicrobial activity. Among different nanostructures, insoluble metallic nanoparticle, soluble non-metallic nanoparticle, graphene-based materials are playing a pivotal roles in the biotechnological applications. This study was carried out to attest the bio-carrying carrying capability of silver, cellulose nanoparticles and singlewalled carbon nanotubes (SWCNT).

Silver nanoparticles have been widely considered to be a vital nanoparticle, produced by different methods, including physical, chemical and biological methods. Physical methods are less preferred under mass scale preparation due to the mandatory of intensive power and consumption of high energy. On the other hand, chemical methods are considered in several cases using polymers, reducing analytical agents, surfactants and protective agents [8]. However, considering the environmental benefits in most of the cases, it has been advisable to avoid the usage of chemical compounds, especially analytical reagents. Because of that, the wise way to produce the silver nanoparticle is through biological method and it was found to be cost-effective and environmentally friendly method to synthesize a stable silver nanoparticle and dominates over other methods [9-11].

On the other hand, cellulose nanoparticles are the non-metallic have been found to have the advantages such as cheaper and consume lesser energy and easier to prepare in large scale to be beneficial to the food and pharmaceutical industries. Cellulose nanoparticle has been prepared by the emulsification processes and applied for the engineering and biomedical purposes [12]. In the biomedical processes, such as targeted drug delivery and treatment cellulose nanoparticles are ideal to use. In addition, the dispersing properties and mechanical strength of cellulose nanoparticle is higher as a polymer [13].

Carbon is the one the most abundant elements in our life and it plays a very important rule in the world such as life creation. There are lot of allotropes contain carbon like diamond (the hardest material), graphite (the softest material) and buckminsterfullerence (ball-like form). Carbon is in group fourth and has atomic number of six in the periodic table. All elements in atomic size cannot be seen by naked eyes and must be observed by the microscope. In atom, there are three particles which are positive charged proton, negative charged electron and neutral charged neutron. The carbon has six protons as the atomic number is six and it has the electronic ground state configuration of $1 s^{2} 2 s^{2} 2 p^{2}$. The graphene-based

\footnotetext{
* Corresponding author: subash@unimap.edu.my
} 
material, SWCNTs can be defined as single-walled of carbon nanotubes appear as a hollow cylinder shape. It is just one atom thick in thickness and can be viewed as a roll. Graphene is a sheet of two-dimension of carbon, atomic scale, honey-comb lattice and it is bounded by $\mathrm{sp}^{2}$ bond (Figure 1). Because of the same element of composition and bond arrangement of SWCNTs and graphene, both having the same properties and characterization. SWCNT has the advantages and their pros in various applications compared to others element [14-15]. Therefore, SWCNTs should be analyzed wisely so that it can give a help in the fields of technology.

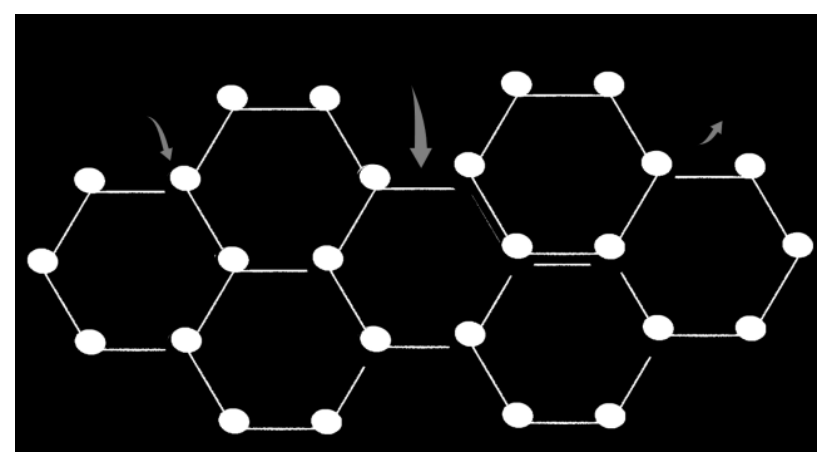

Figure 1. Typical structure of graphene. It has 6 carbon atoms on each ring structure, connected by the molecular bond with the size of $\sim 0.142 \mathrm{~nm}$.

To utilize the above nanomaterials as the base to carry the biological ligands, two herbs were chosen, namely Solanum trilobatum and Hempedu bumi and extracted the compounds from them. S. trilobatum is a commonly used name and also called locally as 'Thoothuvalai', originated from the Asian country, India. This plant is mainly growing in the Southern region of India, which includes the states Tamil Nadu and Kerala and also from some Northern part of India. In addition, this plant is found to grown in Southern part of Asia, including the regions Malaysia, Vietnam and Thailand. The local climate in these parts favour the growth of this plant and appears throughout of the year. S. trilobatum is found to have medicinal value for treating the health issues. Mainly to treat respiratory problems, cough, gastric, asthma, constipation and relieve gas in stomach. $S$. trilobatum has been analysed and noticed to have antimicrobial characteristics, make suitable for the current study.

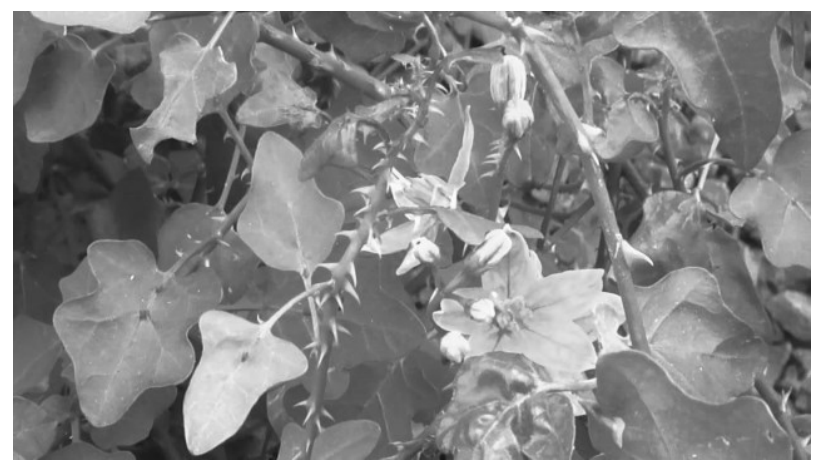

Figure 2. Morphology of Solanum trilobatum.

On the other hand, Hempedu bumi or its scientific name, Andrographis paniculata, is an akin of shrub, belonging to the family of Acanthaceae, also known as Ayurveda Herb (Kalamegha) in India due to its common properties. A. paniculata grows abundantly in India, Taiwan and Sri Lanka. However, it is cultivated extensively in China and Thailand. This plant has long been used in Chinese official herbal medicine against broad spectrum of illness. Past a few decades, $A$. paniculata plant is highly valued by local people as one of the medicine that can treat a variety of diseases. It is highly effective for current global illness and health concerns.

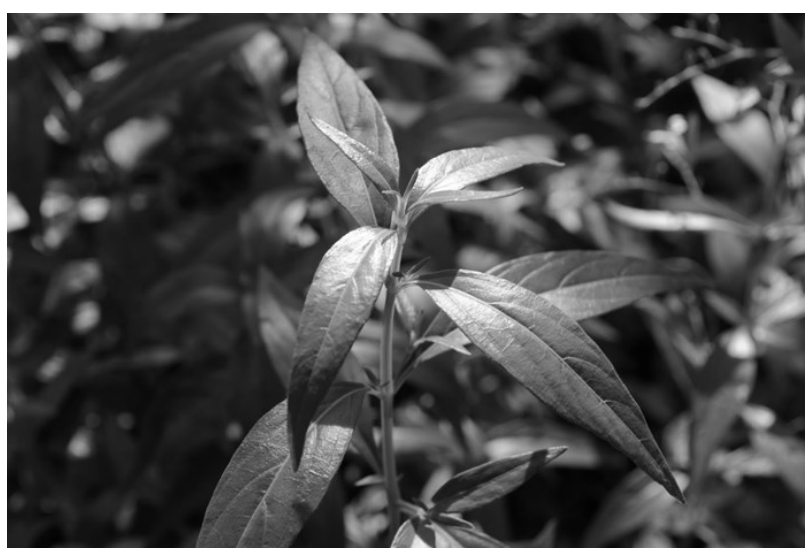

Figure 3. Morphology of Hempedu bumi.

\section{Materials and Methods}

\subsection{Materials}

Silver Nitrate, Sodium Chloride, Tween-20 were purchased from Sigma-Aldrich. Cow urine was collected from the local form in Malaysia. Solanum trilobatum and Hempedu bumi plants were collected from India and Malaysia, respectively. All analytical grade reagents were from Merck. All chemicals and reagents were stored and used as recommended by the supplier. The bacterium, Bacillus sp. was obtained from the culture collection of School of Bioprocess, Universiti Malaysia Perlis, Perlis.

\subsection{Extraction of plant extract}

To prepare the plant extract 5 gram of plant material was soaked in the $50 \mathrm{ml}$ of water for the period of overnight. Next day, the mixture was boiled at $55^{\circ} \mathrm{C}$ for $10 \mathrm{~min}$ and filtered through the filters. Further, these extracts were filtered again using syringe filter (Millipore) having the pore size of $0.45 \mu \mathrm{m}$.

\subsection{Preparation of Nanostructures}


The plant extract of Solanum trilobatum was prepared as mentioned above. Then $1 \mathrm{mM}$ of silver nitrate was mixed with plant extract with the constant stirring. It was continued until the silver nitrate solution become yellowish orange. After the formation of color, the solution was centrifuged at $10,000 \mathrm{xg}$ for $10 \mathrm{~min}$. The obtained pellet was washed with double distilled water twice. For the encapsulation of cow urine, the previous method proposed was followed as outlined [16]. SWCNTs were purchased commercially and conjugated electrostatically with the extract prepared from Hempedu bumi.

\subsection{Scanning Electron Microscope}

All the nanostructures were observed under Field Emission Scanning Electron Microscope (Hitachi, S4300 SE, Japan) with appropriate scanning speed under the applied voltage of $15 \mathrm{kV}$. Magnification was done at 5000x.

\subsection{Biological inhibition assay}

To evaluate the inhibitory activity of the prepared nanostructures conjugated biological ligands, disc diffusion assay was followed. Initially, agar plates using LB media agar were prepared by following the routine procedure. Upon preparation, the culture Bacillus sp. was spread on the agar plate. At the same time, the disc made from the filter paper was placed on the agar plate and loaded with $35 \mu \mathrm{l}$ of the sample to be tested. For the entire test with all samples, the similar volume was used. Ampicillin was used the standard. Then, after continuing the culture at $37^{\circ} \mathrm{C}$ for overnight, the formation of the clear region was measured.

\section{Results and Discussion}

The compounds obtained from the herbal plant have gained a considerable amount of importance in the field of medicine and biotechnology. Several herbal plants are found to have medicinal values and used traditionally for a quite long time to treat illnesses and injuries [17]. In several instances herbal plants have been used directly without further purification steps. However, production of engineered nanomaterials [7] will boost the utilization of herbal compounds by conjugating them on the nanomaterials.

Another nanomaterial is chosen for this study is the cellulose nanoparticle, composed of organic compound with the D-glucose units [18]. Cellulose is one of the cheaper mateials available easily all over the word and about $10^{10}$ to $10^{11}$ tons of the cellulose is annually synthesized and destroyed. Researchers are taking attempts to recycle the cellulose waste in different ways. Currently, cellulose nanoparticle is considered to be the counter for the metallic nanoparticles and play a vital role [19].
Synthesis and fabrication of nanostructures are the currently main streams in the front of nanotechnology in order to engineer the novel nanostructures. These nanostructures have shown to be nanoparticle, nanofilm, nanotube, nanowire, nanowick and combination of these structures. On the other hand, the downstream application of these nanostructures has been focused mainly in the biomedical utilizations. In the field of biomedicine, nanostructures are mainly used to be the carrier for the purpose of interactive analysis and nanodelivery. In the current study, we have chosen three different types of nanostructures, which include silver nanoparticle, cellulose nanoparticle and SWCNT. To demonstrate the suitability of these nanostructures to carry the biological ligands, both plant extract and cow urine were chosen. Plenty of active compounds can be extracted from the plant; on the other hand, cow urine has the effective bioactive compounds (Figure 4).

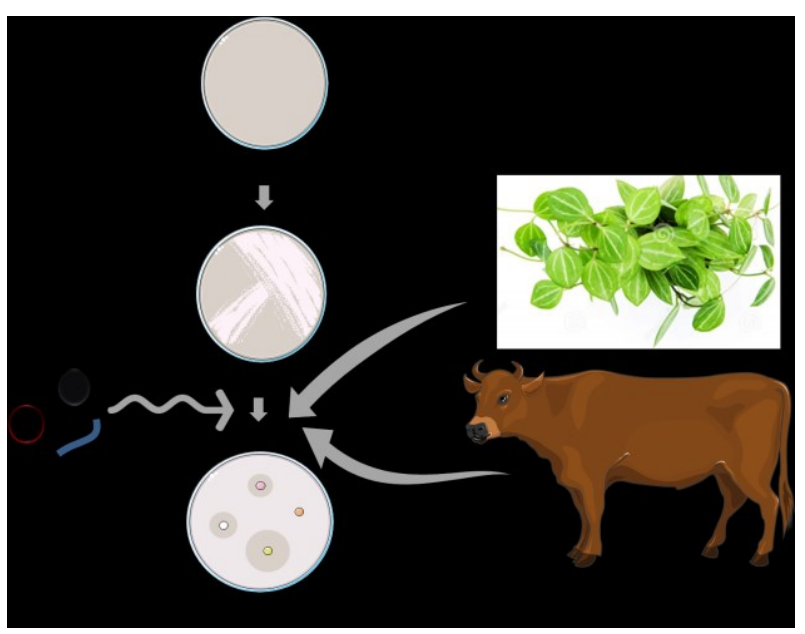

Figure 4. Scheme for the overall study. Either plant extract or cow urine was conjugated on the nanostructures and studied the bacterial inhibition. Bacillus sp. was used as the test bacterium.

\subsection{Silver nanoparticle as the carrier for}

\section{biological ligands}

Biosynthesis production of silver nanoparticles using medicinal plants is more desirable than physical and chemical methods due to its eco-friendliness. The purpose of the present study is to identify the medicinal properties of silver nanoparticles synthesized by herbal extracts. To demonstrate this purpose the compounds from plant 'Solanum trilobatum' were chosen as the capping the reducing agents. Upon completion of the production of silver nanoparticle, it can be considered as it is carrying the biological ligands from S. trilobatum. After synthesizing the silver nanoparticles, they were observed under Field Emission Scanning Electron Microscope (FESEM) as shown in the Figure 5. As per measurement from the FESEM, the size of the produced nanoparticle is estimated to be about $15 \mathrm{~nm}$. Also confirmed by Field Emission Transmission Electron 
Microscope (FETEM; Figure 6). It was also noticed that silver nanoparticles are distributed uniformly. Upon preparation of silver nanoparticles, we evaluated the biological inhibitory activity in order to confirm the carrying of biological ligands. The biological inhibition was compared with the ampicillin $(1 \mathrm{mg} / \mathrm{ml})$ as the standard.

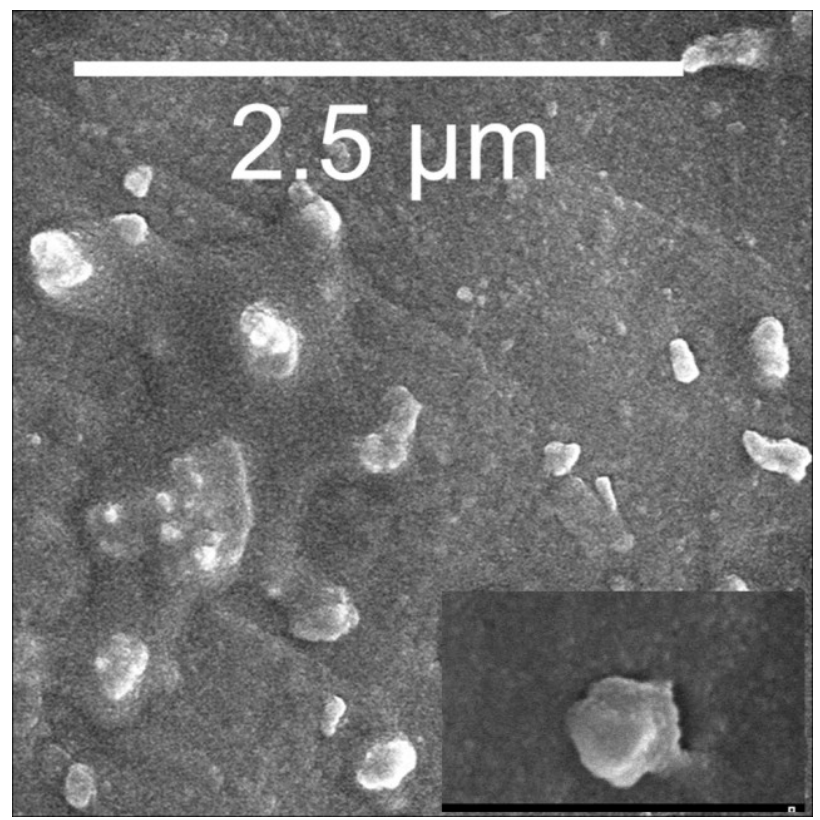

Figure 5. FESEM image of Solanum trilobatum plant extract mediated synthesis of silver nanoparticle. Figure inset display the enlarged view. Performed at $15 \mathrm{kV}$. Scale bar is shown.

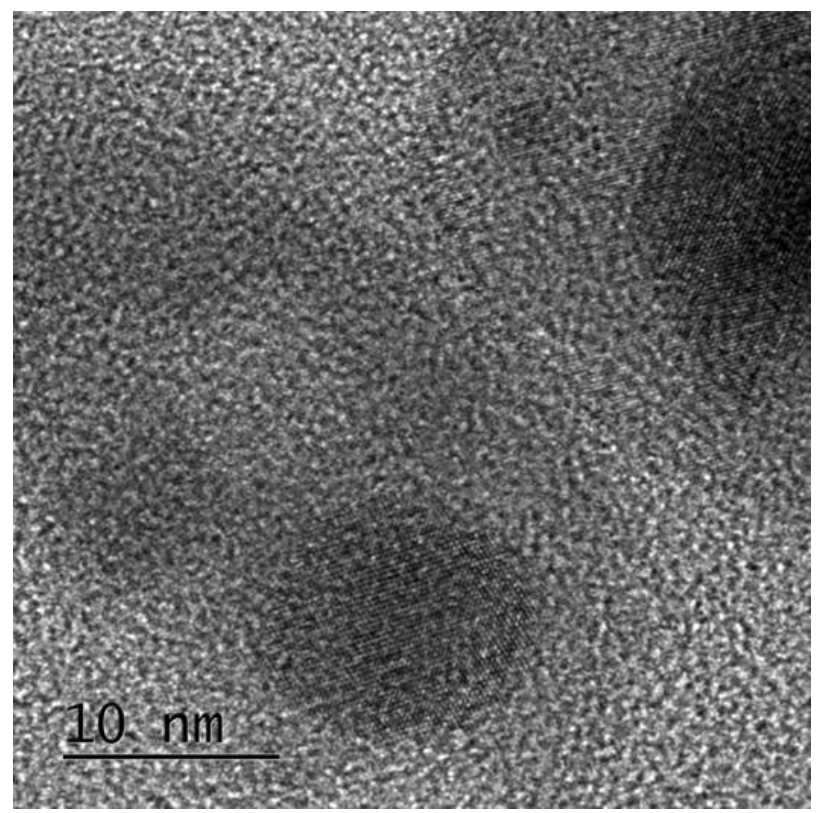

Figure 6. FETEM image of Solanum trilobatum plant extract mediated synthesis of silver nanoparticle. Scale bar is shown.

With the ampicillin using $15 \mu \mathrm{g}$, we could observe the clear zone against the Gram-positive bacterium Bacillus sp. and it shows the clearing region of $15 \pm 0.2 \mathrm{~mm}$. Whereas, S. triblobatum silver nanoparticle has shown he clearing region of $6.5 \pm 0.15 \mathrm{~mm}$ (Figure 7).

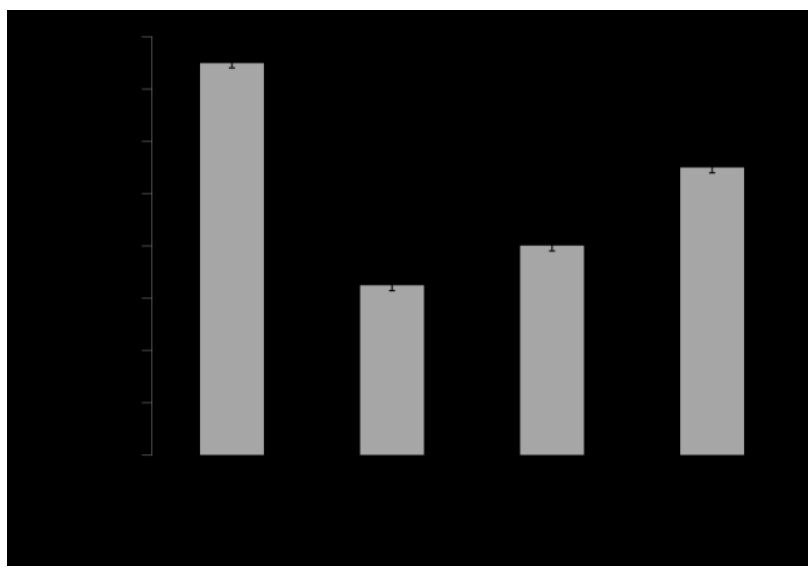

Figure 7. Zone of Inhibition. Measured on the agar plate cultured with Bacillus sp. Disc diffusion assay was followed. Ampicillin was used as the standard solution. Silver and SWCNT were conjugated with the plant extract. Cellulose nanoparticle was encapsulated with cow urine.

By comparison, $S$. triblobatum capped silver nanoparticles have shown nearly half of the inhibition region displayed by $15 \mu \mathrm{g}$ of ampicillin. This analysis confirms the ability of silver nanoparticles to act as the carrier for the biological ligands.

\subsection{Cellulose nanoparticle as the carrier}

\section{for biological ligands}

Similar to the above case with the silver nanoparticle, another nanostructure cellulose nanoparticle was also demonstrated for carrying the biological ligands. In this case, cow urine was collected and encapsulated in the cellulose nanoparticle by microemulsion method as stated earlier [16]. The cow urine encapsulated cellulose nanoparticle was observed under FESEM as displayed in the Figure 8.

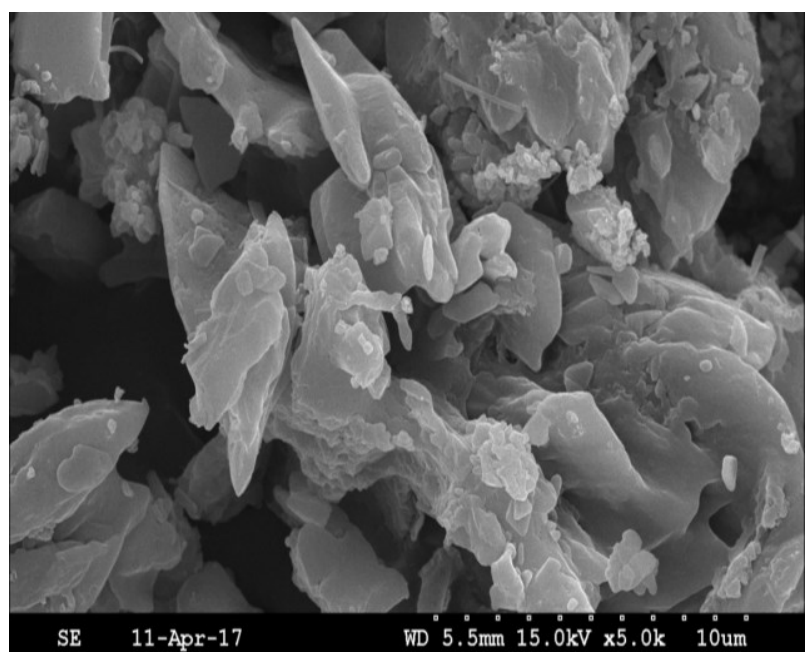


Figure 8. FESEM image of cow urine encapsulated cellulose nanoparticle. Performed at $15 \mathrm{kV}$. Scale bar is shown.

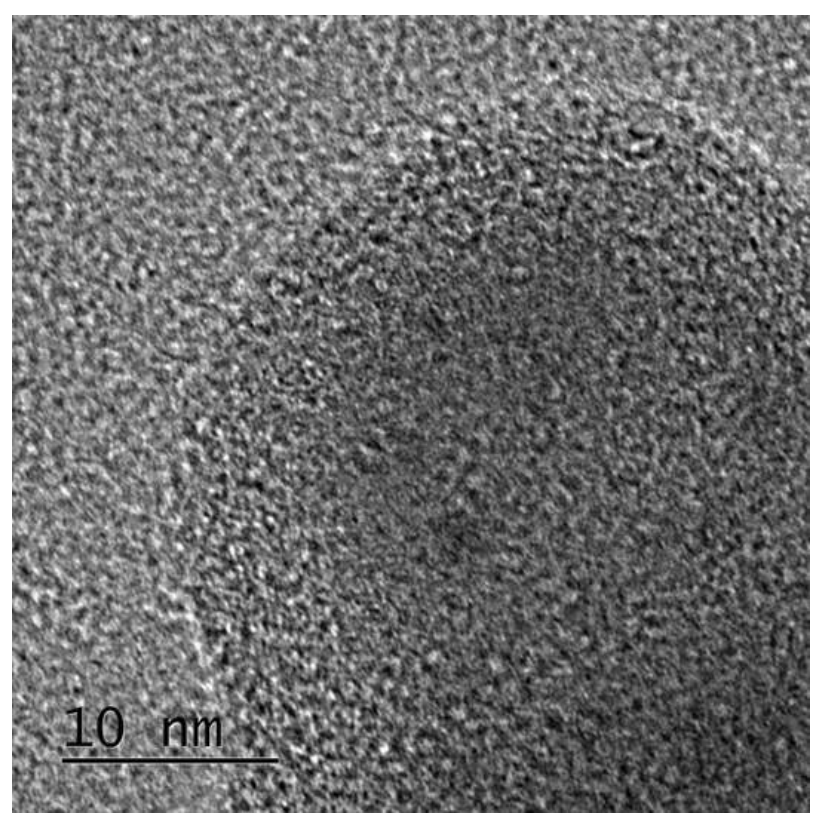

Figure 9. FETEM image of cow urine encapsulated cellulose nanoparticle. Scale bar is shown.

From the FESEM analysis it was noticed that the encapsulated cellulose nanoparticle is agglomerated. This might be due to the soft texture of the cellulose nanoparticle compared to the metallic nanoparticle. This result was further supported by FETEM analysis (Figure 9). As in the case above, we also evaluated the inhibition ability of the cellulose nanoparticle encapsulated cow urine.

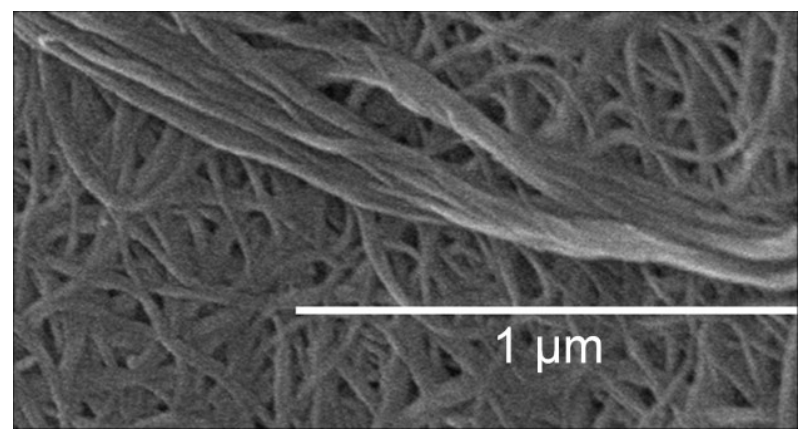

Figure 10. FESEM image of Hempedu bumi plant extract conjugated SWCNTs. The scale bar is shown with $1 \mu \mathrm{m}$ range.

The result was measured to be with the clearing region of $8.0 \pm 0.18 \mathrm{~mm}$ against the bacterium Bacillus sp. (Figure 7). The difference between the clear regions obtained from the above $S$. trilobatum capped silver nanoparticle and cellulose nanoparticle encapsulated cow urine is due to the amount of the biological ligands conjugated and its ability to inhibit the Bacillus sp. In the case of cellulose nanoparticle encapsulation is showed more than half level of inhibition compared to $15 \mu \mathrm{g}$ of ampicillin. This analysis proved as in the case of silver nanoparticle, cellulose nanoparticle can also be the biological carrier.

\subsection{SWCNT as the carrier for biological}

\section{ligands}

In the above cases, we used both metallic and nonmetallic nanoparticles. As the third nanostructure we preferred different nanostructure fabricated based on graphene material, called SWCNTs. The extracted compounds from the medicinally important herb plant Hempedu bumi, was chosen as the biological ligands and conjugated electrostatically on the SWCNTs. SWCNTs were seen under the FESEM and it was obvious with the parallel SWCNTs with the uniform sizes, further SWCNTs were nicely packed (Figure 10). H. bumi extract conjugated SWCNTs was also tested for their inhibitory ability against the bacterium Bacillus sp. Upon measuring, we could found the clear region of 1.1 $\pm 0.19 \mathrm{~mm}$ and this level is close to the level of inhibition obtained with $15 \mu \mathrm{g}$ of ampicillin (Figure 7). This analysis evidenced that the extracted compounds from $H$. bumi conjugated on SWCNT is active with the antibacterial inhibition.

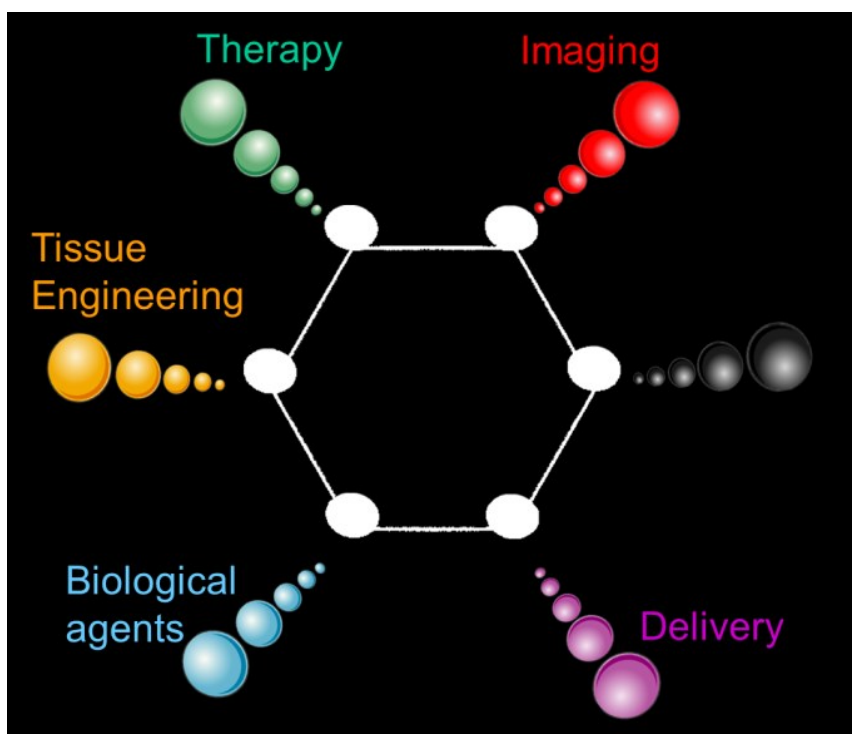

Figure 11. Potential applications of biological ligands conjugated nanomaterials. These applications are highly connected to the field of medicine.

This study clearly demonstrated with different nanomaterials (Silver nanoparticle, Cellulose nanoparticle and SWCNT), for their potential to be active by carrying the biological ligands. Similarly, by mimicking the same strategy for conjugating different biomolecules, it will be highly beneficial for the downstream applications, such as therapy, imaging, 
biosensor, tissue engineering, targeted delivery (Figure 11). As a proof of concept, in the past several studies have been demonstrated for these applications [20-22].

\section{Conclusions}

In the present study, three engineered nanostructures have been chosen, namely silver nanoparticle, cellulose nanoparticle and SWCNT. These nanostructures were conjugated with the biological ligands obtained from the herb $S$. trilobatum, cow urine and the herb H. bumi, respectively. The engineered nanostructures were well formed and uniformly arranged under FESEM analysis. From the inhibition analyses, it was revealed that all these nanostructures have ability to carry the biological ligands. Based on these, in can be concluded that engineered nanostructures are the potential candidates to carry the biological ligands for biomedical applications, especially for pathogenic inhibition.
Drug Targets 18 (2017) 724-733.

15. S. Bharec, R. Kumar, J. Mat. Sci. Mechanical Engg. 2, (2015) 70-73.

16. S.C.B. Gopinath, C.S. Goh, M. Citartan, T. Lakshmipriya, M.K. Arshad, M.F. Fatin, A.R. Ruslinda, U. Hashim, S.V. Chinni, T.H. Tang, Micro Nanosyst. 8, (2016) 1-6.

17. P.S. Latha, K. Kannabiran, African J. Biotechnol. 5 (2006) 2402-2404.

18. S. Kalia, A. Dufresne, B. M. Cherian, B. S. Kaith, L. Avérous, J. Njuguna, E. Nassiopoulos. Int. J. Polymer Sci. 2011 (2011), 837875.

19. M. A. El-Sheikh, S. M. El-Rafie, E. S. AbdelHalim, and M. H. El-Rafie. J. Polymers 2013 (2013), 650837.

20. S.C.B. Gopinath, K. Awazu, M. Fujimaki, K. Shimizu, T. Shima, PLoS One 8 (2013) e69121.

21. S.C.B. Gopinath, P.K.R. Kumar, Acta Biomaterialia 9 (2013) 8932-8941.

22. S.C.B. Gopinath, D. Balasundaresan, J. Akitomi, H. Mizuno, J. Biochem. 140 (2006) 667-676.

\section{References}

1. S. Anniebell, S.C.B. Gopinath, Curr Med Chem. 24 (2017) [Epub ahead of print].

2. K.H.Suk, S.C.B. Gopinath, Curr Med Chem. (2017) [Epub ahead of print].

3. S. Ramanathan, S.C.B. Gopinath, Microsyst. Technol. 23 (2017) 4345-4357.

4. K. Nomura, S.C.B. Gopinath, Nat.Commun. 4 (2013) 2855.

5. M.E. Foo, S.C.B. Gopinath. Biomed. Pharmacotherapy 94 (2017) 354-361.

6. N.Z. Jin, S. Anniebell, S.C.B. Gopinath, Y. Chen, Nanoscale Res. Lett. 11 (2016) 399.

7. A. Suominen, Y. Li, J. Youtie, P. Shapira, J Nanoparticle. 18, (2016) 270.

8. P. Velusamy, C.-H. Su, G. Venkat Kumar, S. Adhikary, K. Pandian, S.C.B. Gopinath, Y.Chen, P. Anbu, PLoS One, 11 (2016) e0157612.

9. S. Ahmed, M. Ahmad, B.L. Swami, S. Ikram, J. Radiation Research and Applied scinces. 9, (2016) 17-28.

10. D. Nayak, S. Ashe, P.R. Rauta, M. Kumari, B. Nayak, Mater. Sci. Eng. C. 58, (2016) 44-52.

11. K. Logaranjan, A.J. Raiza, S.C.B. Gopinath, Y. Chen, K. Pandian, S, Nanoscale Res. Lett. 11 (2016) 520.

12. N. Lin, A. Dufresne, Eur. Poly. J. 59 (2014) 302-325.

13. L. Zhou, H. He, M.C. Li, K. Song, H.N. Cheng, Q. Wu, M, Carbohydr. Polym. 153 (2016) 445-454.

14. D. A. Ghuge, A. R. Shirode, V. J. Kadam. Curr. 